\title{
A Holomorphic Extension Theorem using Clifford Analysis
}

\author{
Ricardo Abreu Blaya • Juan Bory Reyes • \\ Dixan Peña Peña · Frank Sommen
}

Received: 23 May 2009 / Accepted: 7 September 2009 / Published online: 26 September 2009

(C) Birkhäuser Verlag Basel/Switzerland 2009

\begin{abstract}
In this paper a new holomorphic extension theorem is presented using Clifford analysis.
\end{abstract}

Keywords Clifford analysis · Isotonic functions · Holomorphic extension

Mathematics Subject Classification (2000) $\quad 30 \mathrm{G} 35 \cdot 32 \mathrm{~A} 40$

\section{Introduction}

Let $\Omega$ be a simply connected bounded and open set in $\mathbb{C}^{n}$. Suppose that $f$ is a $\mathbb{C}$-valued continuous function on $\partial \Omega$ and let us denote by $M_{1} f$ the Bochner-Martinelli integral

Communicated by Daniel Aron Alpay, Ph.D..

R. Abreu Blaya

Facultad de Informática y Matemática, Universidad de Holguín, 80100 Holguín, Cuba

e-mail: rabreu@facinf.uho.edu.cu

J. Bory Reyes

Departamento de Matemática, Universidad de Oriente, 90500 Santiago de Cuba, Cuba e-mail: jbory@rect.uo.edu.cu

D. Peña Peña $(\varangle)$

Department of Mathematics, Aveiro University, 3810-193 Aveiro, Portugal

e-mail: dixanpena@ua.pt; dixanpena@gmail.com

F. Sommen

Department of Mathematical Analysis, Ghent University,

Galglaan 2, 9000 Ghent, Belgium

e-mail: fs@cage.UGent.be 
of function $f$. It is a well-known fact that $M_{1} f$ is a harmonic function in $\mathbb{C}^{n} \backslash \partial \Omega$ and vanishes at infinity.

Aronov and Kytmanov provided in [3] (see also [21,22]) the following characterizations:

Theorem 1.1 If $\partial \Omega$ is a smooth surface and $f$ is a continuously differentiable function in $\partial \Omega$, then a necessary and sufficient condition for $f$ to have a holomorphic extension to $\Omega$ is that $M_{1} f(\underline{x})=0$ for $\underline{x} \in \mathbb{C}^{n} \backslash \bar{\Omega}$.

Theorem 1.2 If $\partial \Omega$ is a piecewise smooth surface and $F$ is a continuously differentiable function in $\bar{\Omega}$, then $F$ is holomorphic in $\Omega$ if and only if $F(\underline{x})=M_{1} F(\underline{x})$ for $\underline{x} \in \Omega$.

The aim of this paper is to present alternative characterizations using Clifford analysis, the latter being a direct and elegant generalization to higher dimension of the theory of holomorphic functions in the complex plane. We will also re-establish the results of Aronov and Kytmanov for the particular case $n=2$ using our techniques.

The outline of the paper is as follows. For the reader who is not familiar with Clifford analysis, we recall some of its basics in Sect. 2. In Sect. 3 we introduce our main techniques of work: the isotonic functions and the isotonic Cauchy type integral. Section 4 contains a brief summary of the holomorphic functions. In the last two sections our main results are stated and proved.

\section{Some Basic Notions of Clifford Analysis}

Clifford analysis (see, e.g. $[5,9,17]$ ) offers a function theory which is a higher dimensional analogue of the theory of the holomorphic functions of one complex variable.

The functions considered are defined in the Euclidean space $\mathbb{R}^{m}(m>1)$ and take their values in the complex Clifford algebra $\mathbb{C}_{m}$. Let $\left(e_{1}, \ldots, e_{m}\right)$ be an orthonormal basis of $\mathbb{R}^{m}$, then a basis for the Clifford algebra $\mathbb{C}_{m}$ is given by $\left(e_{A}: A \subset\{1, \ldots, m\}\right)$, where $e_{\emptyset}=1$ is the identity element, $e_{\{j\}}=e_{j}, j=1, \ldots, m$ and $e_{A}=e_{j_{1}} \cdots e_{j_{k}}$, $A=\left\{j_{1}, \ldots, j_{k}\right\}$ being ordered such that $j_{1}<\cdots<j_{k}$. Any Clifford number $a \in \mathbb{C}_{m}$ may thus be written as

$$
a=\sum_{A} a_{A} e_{A}, \quad a_{A} \in \mathbb{C} .
$$

The non-commutative multiplication in $\mathbb{C}_{m}$ is governed by the rules

$$
\begin{aligned}
& e_{j}^{2}=-1, \quad j=1, \ldots, m, \\
& e_{j} e_{k}+e_{k} e_{j}=0, \quad 1 \leq j \neq k \leq m .
\end{aligned}
$$


The main involution $a \rightarrow \tilde{a}$ and the conjugation $a \rightarrow \bar{a}$ are respectively given by

$$
\begin{aligned}
& \tilde{a}=\sum_{A} a_{A} \tilde{e}_{A}, \quad \tilde{e}_{A}=(-1)^{k} e_{A} \quad \text { if } \quad|A|=k, \\
& \bar{a}=\sum_{A} \bar{a}_{A} \bar{e}_{A}, \quad \bar{e}_{A}=(-1)^{\frac{k(k+1)}{2}} e_{A} \quad \text { if } \quad|A|=k .
\end{aligned}
$$

One easily checks that

$$
\begin{aligned}
& \tilde{a b}=\tilde{a} \tilde{b}, \\
& \overline{a b}=\bar{b} \bar{a},
\end{aligned}
$$

for any $a, b \in \mathbb{C}_{m}$.

For each $k \in\{0,1, \ldots, m\}$, we call

$$
\mathbb{C}_{m}^{(k)}=\left\{a \in \mathbb{C}_{m}: a=\sum_{|A|=k} a_{A} e_{A}\right\}
$$

the subspace of $k$-vectors, i.e. the space spanned by the products of $k$ different basis vectors. In this way we have that

$$
\mathbb{C}_{m}=\bigoplus_{k=0}^{m} \mathbb{C}_{m}^{(k)}
$$

and hence for any $a \in \mathbb{C}_{m}$

$$
a=\sum_{k=0}^{m}[a]_{k}
$$

where $[.]_{k}$ is the projection operator on $\mathbb{C}_{m}^{(k)}$.

In particular, the 0 -vectors and 1 -vectors are simply called scalars and vectors respectively. If we identify the $\mathbb{R}^{m}$-vector $\left(x_{1}, \ldots, x_{m}\right)$ with the real Clifford vector $\underline{x}=\sum_{j=1}^{m} x_{j} e_{j}$, then $\mathbb{R}^{m}$ may be considered as a subspace of $\mathbb{C}_{m}$.

By means of the conjugation, a norm $|a|$ may be defined for each $a \in \mathbb{C}_{m}$ by putting

$$
|a|^{2}=[a \bar{a}]_{0}=\sum_{A}\left|a_{A}\right|^{2}
$$

The product of two Clifford vectors $\underline{x}=\sum_{j=1}^{m} x_{j} e_{j}$ and $\underline{y}=\sum_{j=1}^{m} y_{j} e_{j}$ splits into a scalar part and a 2 -vector or so-called bivector part

$$
\underline{x} \underline{y}=\underline{x} \bullet \underline{y}+\underline{x} \wedge \underline{y}
$$


where

$$
\underline{x} \bullet \underline{y}=-\langle\underline{x}, \underline{y}\rangle=-\sum_{j=1}^{m} x_{j} y_{j}
$$

equals, up to a minus sign, the standard Euclidean inner product between $\underline{x}$ and $\underline{y}$, while

$$
\underline{x} \wedge \underline{y}=\sum_{j=1}^{m} \sum_{k=j+1}^{m} e_{j} e_{k}\left(x_{j} y_{k}-x_{k} y_{j}\right)
$$

represents the standard outer (or wedge) product between them.

The elliptic vector differential operator of the first order

$$
\partial_{\underline{x}}=\sum_{j=1}^{m} e_{j} \partial_{x_{j}}
$$

called Dirac operator, factorizes the Laplace operator in $\mathbb{R}^{m}$, i.e.

$$
\Delta_{\underline{x}}=\sum_{j=1}^{m} \partial_{x_{j}}^{2}=-\partial_{\underline{x}}^{2} .
$$

The main objects of study in Clifford analysis are the so-called monogenic functions which may be described as null solutions of the Dirac operator $\partial_{\underline{x}}$. More precisely, a function $f(\underline{x})$ defined and continuously differentiable in an open set $\Omega$ of $\mathbb{R}^{m}$ and taking values in $\mathbb{C}_{m}$, is called a (left) monogenic function in $\Omega$ if and only if it fulfills in $\Omega$ the equation

$$
\partial_{\underline{x}} f \equiv \sum_{j=1}^{m} \sum_{A} e_{j} e_{A} \partial_{x_{j}} f_{A}=0 .
$$

From (2.2) it follows that any monogenic function in $\Omega$ is harmonic in $\Omega$ and hence real-analytic in $\Omega$.

\section{Isotonic Functions and the Isotonic Cauchy Type Integral}

A special type of monogenic functions arises when the dimension of $\mathbb{R}^{m}$ is even. Let $m=2 n$ and denote by $\mathbb{C}_{n}$ the complex Clifford algebra generated by $\left(e_{1}, \ldots, e_{n}\right)$. Next, we introduce the primitive idempotent

$$
I=\prod_{j=1}^{n} I_{j}
$$


with

$$
I_{j}=\frac{1}{2}\left(1+i e_{j} e_{n+j}\right), \quad j=1, \ldots, n .
$$

The following conversion relations hold

$$
e_{n+j} I=i e_{j} I, \quad j=1, \ldots, n,
$$

and for $a \in \mathbb{C}_{n}$ we also have that

$$
a I=0 \quad \Leftrightarrow \quad a=0 .
$$

Below, we will need the following Clifford vectors and their corresponding Dirac operators:

$$
\begin{aligned}
& \underline{x}_{1}=\sum_{j=1}^{n} x_{j} e_{j}, \quad \partial_{\underline{x}_{1}}=\sum_{j=1}^{n} e_{j} \partial_{x_{j}}, \\
& \underline{x}_{2}=\sum_{j=1}^{n} x_{n+j} e_{j}, \quad \partial_{\underline{x}_{2}}=\sum_{j=1}^{n} e_{j} \partial_{x_{n+j}} .
\end{aligned}
$$

Now consider a Clifford vector $\underline{x}$ which may be written as

$$
\underline{x}=\sum_{j=1}^{n}\left(x_{j} e_{j}+x_{n+j} e_{n+j}\right)
$$

For $a \in \mathbb{C}_{n}$ it follows that

$$
\underline{x} a I=\underline{x}_{1} a I+\sum_{j=1}^{n} x_{n+j} e_{n+j} a I=\underline{x}_{1} a I+\tilde{a} \sum_{j=1}^{n} x_{n+j} e_{n+j} I,
$$

whence application of (3.1) yields

$$
\underline{x} a I=\left(\underline{x}_{1} a+i \tilde{a} \underline{x}_{2}\right) I .
$$

If we now take a continuously differentiable function $f: \Omega \subset \mathbb{R}^{2 n} \rightarrow \mathbb{C}_{n}$, then we learn from (3.3) that

$$
\partial_{\underline{x}}(f I)=\left(\partial_{\underline{x}_{1}} f+i \tilde{f} \partial_{\underline{x}_{2}}\right) I,
$$

whence it follows from (3.2) that the spinor-valued function $f I$ is monogenic if and only if

$$
\partial_{\underline{x}_{1}} f+i \tilde{f} \partial_{\underline{x}_{2}}=0 \text {. }
$$


Definition 3.1 A function $f$ defined and continuously differentiable in an open set $\Omega$ of $\mathbb{R}^{2 n}$ with values in $\mathbb{C}_{n}$, which satisfies in $\Omega$ the above equation, is said to be isotonic in $\Omega$ (see [33]).

Note that an isotonic function is also harmonic. This may be easily proved using (3.2) as well as the equalities

$$
0=\Delta_{\underline{x}}(f I)=\left(\Delta_{\underline{x}} f\right) I .
$$

The isotonic functions are closely related with the so-called Hermitean monogenic functions, which are a recent refinement of the monogenic functions (see $[6,30,31]$ ). The connection is given as follows: $f I$ is Hermitean monogenic if and only if $[f]_{k}$ is isotonic for $k=0, \ldots, n$.

It is worth noting that if in particular $f$ takes values in the space of scalars $\mathbb{C}$, then $f$ is isotonic if and only if

$$
\left(\partial_{x_{j}}+i \partial_{x_{n+j}}\right) f=0, \quad j=1, \ldots, n
$$

which means that $f$ is a holomorphic function in the complex variables $z_{j}=x_{j}+$ $i x_{n+j}, j=1, \ldots, n$.

Throughout the paper, $\Omega^{+}$stands for a simply connected bounded and open set in $\mathbb{R}^{2 n}$ with boundary $\Sigma$ such that $\mathcal{H}^{2 n-1}(\Sigma)<\infty$. Here $\mathcal{H}^{2 n-1}$ denotes the $(2 n-1)$-dimensional Hausdorff measure (see $[10,11,25])$. Let $\Omega^{-}=\mathbb{R}^{2 n} \backslash \overline{\Omega^{+}}$.

The open ball of radius $\epsilon>0$ centered at a point $\underline{x}$ in $\mathbb{R}^{2 n}$ will be denoted by $B(\underline{x}, \epsilon)$ and is defined by

$$
B(\underline{x}, \epsilon)=\left\{\underline{y} \in \mathbb{R}^{2 n}:|\underline{y}-\underline{x}|<\epsilon\right\} .
$$

Put $d=\sup \{|\underline{x}-\underline{y}|: \underline{x}, y \in \Sigma\}$. Let $\theta_{z}(\epsilon)=\mathcal{H}^{2 n-1}(\Sigma \cap B(\underline{z}, \epsilon))$ for $\underline{z} \in \Sigma$ and $\epsilon>0$. The surface $\Sigma$ is called Ahlfors-David-regular (AD-regular) if there exists a constant $C>0$ such that

$$
C^{-1} \epsilon^{2 n-1} \leq \theta_{\underline{z}}(\epsilon) \leq C \epsilon^{2 n-1}
$$

for all $\underline{z} \in \Sigma$ and $0<\epsilon \leq d$ (see [8]).

We denote by

$$
\underline{v}(\underline{y})=\sum_{j=1}^{n}\left(v_{j}(\underline{y}) e_{j}+v_{n+j}(\underline{y}) e_{n+j}\right)
$$

the exterior unit normal vector on $\Sigma$ at the point $\underline{y}$ introduced by Federer (see [11]). Let

$$
\underline{v}_{1}(\underline{y})=\sum_{j=1}^{n} v_{j}(\underline{y}) e_{j} \text { and } \underline{v}_{2}(\underline{y})=\sum_{j=1}^{n} v_{n+j}(\underline{y}) e_{j}, \quad \underline{y} \in \Sigma .
$$


The modulus of continuity of a continuous function $f$ on $\Sigma$ will be denoted by $\omega_{f}$ and is defined by

$$
\omega_{f}(\tau)=\tau \sup _{\delta \geq \tau} \delta^{-1} \sup _{\left|\underline{z}_{1}-\underline{z}_{2}\right| \leq \delta}\left|f\left(\underline{z}_{1}\right)-f\left(\underline{z}_{2}\right)\right|, \quad \tau \in(0, d] .
$$

A function $\varphi:(0, d] \rightarrow \mathbb{R}_{+}$with $\varphi(0+)=0$ is said to be a majorant if $\varphi(\tau)$ is non-decreasing and $\varphi(\tau) / \tau$ is non-increasing for $\tau \in(0, d]$.

Let us denote by $H_{\varphi}(\Sigma)$ the set of continuous functions $f$ on $\Sigma$ satisfying a generalized Hölder condition, i.e.

$$
\left|f\left(\underline{z}_{1}\right)-f\left(\underline{z}_{2}\right)\right| \leq C \varphi\left(\left|\underline{z}_{1}-\underline{z}_{2}\right|\right), \quad \underline{z}_{1}, \underline{z}_{2} \in \Sigma,
$$

or equivalently

$$
\omega_{f}(\tau) \leq C \varphi(\tau), \quad \tau \in(0, d]
$$

where $\varphi$ is a majorant and $C$ is a positive constant.

It is evident that for $\varphi(\tau)=\tau^{\alpha}, 0<\alpha \leq 1, H_{\varphi}(\Sigma)$ is nothing else but the classical set of Hölder continuous functions $C^{0, \alpha}(\bar{\Sigma})$. If $\alpha=1$, then the function $f$ satisfies a Lipschitz condition.

If moreover for a majorant $\varphi$ there exists a constant $C>0$ such that

$$
\int_{0}^{\epsilon} \frac{\varphi(\tau)}{\tau} d \tau+\epsilon \int_{\epsilon}^{d} \frac{\varphi(\tau)}{\tau^{2}} d \tau \leq C \varphi(\epsilon), \quad \epsilon \in(0, d]
$$

then $\varphi$ is said to be a regular majorant (see [18]). Note that $\varphi(\tau)=\tau^{\alpha}, 0<\alpha<1$, is a regular majorant.

Let $f$ be a $\mathbb{C}_{n}$-valued continuous function on $\Sigma$. The isotonic Cauchy type integral of $f$ will be denoted by $\mathrm{C}_{\Sigma}^{\text {isot }} f$ and defined by

$$
\begin{aligned}
\mathrm{C}_{\Sigma}^{\mathrm{isot}} f(\underline{x})= & \int_{\Sigma}\left(E_{1}(\underline{y}-\underline{x})\left(\underline{v}_{1}(\underline{y}) f(\underline{y})+i \tilde{f}(\underline{y}) \underline{\underline{v}}_{2}(\underline{y})\right)\right. \\
& \left.+\left(f(\underline{y}) \underline{\underline{y}}_{2}(\underline{y})-i \underline{\underline{y}}_{1}(\underline{y}) \tilde{f}(\underline{y})\right) E_{2}(\underline{y}-\underline{x})\right) d \mathcal{H}^{2 n-1}(\underline{y}), \quad \underline{x} \in \mathbb{R}^{2 n} \backslash \Sigma,
\end{aligned}
$$

where

$$
E_{1}(\underline{x})=-\frac{1}{\omega_{2 n}} \frac{\underline{x}_{1}}{|\underline{x}|^{2 n}} \quad \text { and } \quad E_{2}(\underline{x})=-\frac{1}{\omega_{2 n}} \frac{\underline{x}_{2}}{|\underline{x}|^{2 n}}, \quad \underline{x} \in \mathbb{R}^{2 n} \backslash\{0\},
$$

and $\omega_{2 n}$ is the area of the unit sphere $S^{2 n-1}$ in $\mathbb{R}^{2 n}$. It easily follows that $C_{\Sigma}^{\text {isot }} f$ is isotonic in $\mathbb{R}^{2 n} \backslash \Sigma$ and vanishes at infinity. 
We now define the isotonic singular integral operator of $f$ as

$$
\mathrm{S}_{\Sigma}^{\mathrm{isot}} f(\underline{z})=2 \lim _{\epsilon \rightarrow 0+} \mathrm{S}_{\Sigma, \epsilon}^{\mathrm{isot}} f(\underline{z})+f(\underline{z}), \quad \underline{z} \in \Sigma,
$$

where $\mathrm{S}_{\Sigma, \epsilon}^{\text {isot }} f$ denotes the truncated integral defined by

$$
\begin{aligned}
\mathrm{S}_{\Sigma, \epsilon}^{\text {isot }} f(\underline{z})= & \int_{\Sigma \backslash B(\underline{z}, \epsilon)}\left(E_{1}(\underline{y}-\underline{z})\left(\underline{v}_{1}(\underline{y})(f(\underline{y})-f(\underline{z}))+i(\tilde{f}(\underline{y})-\tilde{f}(\underline{z})) \underline{v}_{2}(\underline{y})\right)\right. \\
& \left.+\left((f(\underline{y})-f(\underline{z})) \underline{v}_{2}(\underline{y})-i \underline{v}_{1}(\underline{y})(\tilde{f}(\underline{y})-\tilde{f}(\underline{z}))\right) E_{2}(\underline{y}-\underline{z})\right) d \mathcal{H}^{2 n-1}(\underline{y}) .
\end{aligned}
$$

The following results will be useful in the paper. For the proof of Theorems 3.2, 3.3 and 3.4 we refer the reader to $[1,4,33]$

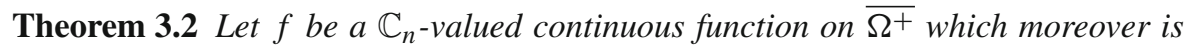
isotonic in $\Omega^{+}$. Then

$$
\mathrm{C}_{\Sigma}^{\text {isot }} f(\underline{x})= \begin{cases}f(\underline{x}) & \text { for } \\ 0 & \text { for } \underline{x} \in \Omega^{+} \\ & \underline{x}\end{cases}
$$

Theorem 3.3 Assume that $\Sigma$ is an AD-regular surface and that $f$ is a $\mathbb{C}_{n}$-valued function which belongs to $H_{\varphi}(\Sigma)$, where $\varphi$ is a regular majorant. Then $\mathrm{C}_{\Sigma}^{\text {isot }} f$ has continuous limit values on $\Sigma$ given by

$$
\lim _{\Omega^{ \pm} \ni \underline{x} \rightarrow \underline{z}} \mathrm{C}_{\Sigma}^{\text {isot }} f(\underline{x})=\frac{1}{2}\left(\mathrm{~S}_{\Sigma}^{\text {isot }} f(\underline{z}) \pm f(\underline{z})\right), \quad \underline{z} \in \Sigma .
$$

Theorem 3.4 Assume that $\Sigma$ is an AD-regular surface and let $\varphi$ be a regular majorant. Then the isotonic singular integral operator $S_{\Sigma}^{\text {isot }}$ is a bounded operator mapping $H_{\varphi}(\Sigma)$ into itself. Moreover, the formula

$$
\left(\mathrm{S}_{\Sigma}^{\text {isot }}\right)^{2} f=f
$$

holds for all $f \in H_{\varphi}(\Sigma)$, i.e. $\mathrm{S}_{\Sigma}^{\text {isot }}$ is an involution on $H_{\varphi}(\Sigma)$.

Lemma 3.5 Let $f$ be a $\mathbb{C}_{n}$-valued continuous function on $\Sigma, \underline{z} \in \Sigma$ and $\epsilon>0$.

(i) If $\underline{x} \in \Omega^{+}$is such that $|\underline{x}-\underline{z}|=\epsilon / 2$, then we have that

$$
\begin{aligned}
& \left|\mathrm{C}_{\Sigma}^{\text {isot }} f(\underline{x})-\mathrm{S}_{\Sigma, \epsilon}^{\mathrm{isot}} f(\underline{z})-f(\underline{z})\right| \\
& \quad \leq C\left(\frac{\theta_{\underline{z}}(\epsilon)}{(\operatorname{dist}(\underline{x}, \Sigma))^{2 n-1}} \omega_{f}(\epsilon)+\epsilon \int_{\epsilon}^{d} \frac{\omega_{f}(\tau)}{\tau^{2 n}} d \theta_{\underline{z}}(\tau)\right) .
\end{aligned}
$$


(ii) If $\underline{x} \in \Omega^{-}$is such that $|\underline{x}-\underline{z}|=\epsilon / 2$, then we have that

$$
\begin{aligned}
& \left|\mathrm{C}_{\Sigma}^{\text {isot }} f(\underline{x})-\mathrm{S}_{\Sigma, \epsilon}^{\text {isot }} f(\underline{z})\right| \\
& \quad \leq C\left(\frac{\theta_{\underline{z}}(\epsilon)}{(\operatorname{dist}(\underline{x}, \Sigma))^{2 n-1}} \omega_{f}(\epsilon)+\epsilon \int_{\epsilon}^{d} \frac{\omega_{f}(\tau)}{\tau^{2 n}} d \theta_{\underline{z}}(\tau)\right) .
\end{aligned}
$$

Proof Let $\underline{x} \in \Omega^{+}$with $|\underline{x}-\underline{z}|=\epsilon / 2$. Then

$$
\begin{aligned}
\mathrm{C}_{\Sigma}^{\text {isot }} & f(\underline{x})-\mathrm{S}_{\Sigma, \epsilon}^{\mathrm{isot}} f(\underline{z})-f(\underline{z}) \\
= & \int_{\Sigma \cap B(\underline{z}, \epsilon)}\left(E_{1}(\underline{y}-\underline{x})\left(\underline{v}_{1}(\underline{y})(f(\underline{y})-f(\underline{z}))+i\left(\tilde{f}(\underline{y})-\tilde{f}\left(\underline{z}^{\prime}\right)\right) \underline{v}_{2}(\underline{y})\right)\right. \\
& \left.+\left((f(\underline{y})-f(\underline{z})) \underline{v}_{2}(\underline{y})-i \underline{v}_{1}(\underline{y})(\tilde{f}(\underline{y})-\tilde{f}(\underline{z}))\right) E_{2}(\underline{y}-\underline{x})\right) d \mathcal{H}^{2 n-1}(\underline{y}) \\
& +\int_{\Sigma \backslash B(\underline{z}, \epsilon)}\left(( E _ { 1 } ( \underline { y } - \underline { x } ) - E _ { 1 } ( \underline { y } - \underline { z } ) ) \left(\underline{v}_{1}(\underline{y})(f(\underline{y})-f(\underline{z}))\right.\right. \\
& \left.+i(\tilde{f}(\underline{y})-\tilde{f}(\underline{z})) \underline{v}_{2}(\underline{y})\right) \\
& +\left((f(\underline{y})-f(\underline{z})) \underline{v}_{2}(\underline{y})-i \underline{v}_{1}(\underline{y})(\tilde{f}(\underline{y})-\tilde{f}(\underline{z}))\right) \\
& \left.\times\left(E_{2}(\underline{y}-\underline{x})-E_{2}(\underline{y}-\underline{z})\right)\right) d \mathcal{H}^{2 n-1}(\underline{y}) .
\end{aligned}
$$

Let us denote by $I_{1}$ and $I_{2}$ the integrals on the right-hand side of the previous equality. For $I_{1}$ we obtain

$$
\begin{aligned}
\left|I_{1}\right| & \leq C \int_{\Sigma \cap B(\underline{z}, \epsilon)} \frac{|f(\underline{y})-f(\underline{z})|}{|\underline{y}-\underline{x}|^{2 n-1}} d \mathcal{H}^{2 n-1}(\underline{y}) \\
& \leq C \int_{\Sigma \cap B(\underline{z}, \epsilon)} \frac{\omega_{f}(\underline{\mid y}-\underline{z} \mid)}{|\underline{y}-\underline{x}|^{2 n-1}} d \mathcal{H}^{2 n-1}(\underline{y}) \\
& \leq C \frac{\omega_{f}(\epsilon)}{(\operatorname{dist}(\underline{x}, \Sigma))^{2 n-1}} \int_{\Sigma \cap B(\underline{z}, \epsilon)} d \mathcal{H}^{2 n-1}(\underline{y}) \\
& =C \frac{\theta_{\underline{z}}(\epsilon)}{(\operatorname{dist}(\underline{x}, \Sigma))^{2 n-1}} \omega_{f}(\epsilon) .
\end{aligned}
$$

To estimate $I_{2}$, we note that

$$
\left|E_{k}(\underline{y}-\underline{x})-E_{k}(\underline{y}-\underline{z})\right| \leq C|\underline{x}-\underline{z}| \sum_{j=0}^{2 n-1} \frac{1}{|\underline{y}-\underline{x}|^{2 n-j}|\underline{y}-\underline{z}|^{j}}, \quad k=1,2 .
$$


Now for $\underline{y} \in \Sigma \backslash B(\underline{z}, \epsilon)$ we have that

$$
\epsilon \leq|\underline{y}-\underline{z}| \leq|\underline{y}-\underline{x}|+|\underline{x}-\underline{z}|=|\underline{y}-\underline{x}|+\epsilon / 2,
$$

and therefore $|\underline{y}-\underline{z}| \leq 2|\underline{y}-\underline{x}|$. It follows that

$$
\left|E_{k}(\underline{y}-\underline{x})-E_{k}(\underline{y}-\underline{z})\right| \leq C \frac{|\underline{x}-\underline{z}|}{|\underline{y}-\underline{z}|^{2 n}}, \quad \underline{y} \in \Sigma \backslash B(\underline{z}, \epsilon), k=1,2 .
$$

We thus get

$$
\left|I_{2}\right| \leq C|\underline{x}-\underline{z}| \int_{\Sigma \backslash B(\underline{z}, \epsilon)} \frac{\omega_{f}(|\underline{y}-\underline{z}|)}{|\underline{y}-\underline{z}|^{2 n}} d \mathcal{H}^{2 n-1}(\underline{y}) \leq C \epsilon \int_{\epsilon}^{d} \frac{\omega_{f}(\tau)}{\tau^{2 n}} d \theta_{\underline{z}}(\tau),
$$

which completes the proof of (i). In a similar way we can prove (ii).

\section{Holomorphic Functions}

Let $m=2 n$. We shall here identify $\mathbb{R}^{2 n}$ with $\mathbb{C}^{n}$ by associating to any element $\left(x_{1}, \ldots, x_{2 n}\right) \in \mathbb{R}^{2 n}$ the complex vector $\left(z_{1}, \ldots, z_{n}\right) \in \mathbb{C}^{n}$ with $z_{j}=x_{j}+i x_{n+j}$, $j=1, \ldots, n$.

The theory of several complex variables is a natural extension of classical complex analysis to the multivariable setting. For a detailed treatment we refer the reader to, e.g. $[16,19,20,27]$.

A continuous function $f: \Omega \rightarrow \mathbb{C}$ on an open set $\Omega$ in $\mathbb{R}^{2 n}$ is said to be holomorphic in $\Omega$ if $f$ is holomorphic in each variable $z_{j}(j=1, \ldots, n)$ separately, i.e. if it satisfies in $\Omega$ the Cauchy-Riemann equations

$$
\left(\partial_{x_{j}}+i \partial_{x_{n+j}}\right) f=0, \quad j=1, \ldots, n
$$

Many basic results of classical one variable complex analysis generalize in a natural way to several variables. However, also new and surprising phenomena emerge, an example of which is given in the following lemma (see [28]).

Lemma 4.1 Suppose that $K$ is a compact subset of $\mathbb{R}^{2 n}, n \geq 2$, such that $\mathbb{R}^{2 n} \backslash K$ is connected. If $f$ is holomorphic and bounded in $\mathbb{R}^{2 n} \backslash K$, then $f$ is a constant.

Proof By Hartogs' theorem, we have that $f$ may be uniquely extended to a holomorphic function in $\mathbb{R}^{2 n}$. Clearly, this extension is bounded in $\mathbb{R}^{2 n}$ and therefore is a constant by Liouville's theorem. 


\section{Holomorphic Extension for Hölder Continuous Functions}

Let $f$ be a $\mathbb{C}$-valued continuous function on $\Sigma$. The Bochner-Martinelli integral (see [21] and the references given there) is defined by

$$
M_{1} f(\underline{x})=-\int_{\Sigma}\left\langle E_{1}(\underline{y}-\underline{x})-i E_{2}(\underline{y}-\underline{x}), \underline{v}_{1}(\underline{y})+i \underline{v}_{2}(\underline{y})\right\rangle f(\underline{y}) d \mathcal{H}^{2 n-1}(\underline{y}),
$$

$\underline{x} \in \mathbb{R}^{2 n} \backslash \Sigma$.

On account of (2.1), we have that

$$
\mathrm{C}_{\Sigma}^{\text {isot }} f(\underline{x})=M_{1} f(\underline{x})+M_{2} f(\underline{x}), \quad \underline{x} \in \mathbb{R}^{2 n} \backslash \Sigma,
$$

where $M_{2}$ is a bivector-valued integral operator given by

$$
M_{2} f(\underline{x})=\int_{\Sigma}\left(E_{1}(\underline{y}-\underline{x})+i E_{2}(\underline{y}-\underline{x})\right) \wedge\left(\underline{v}_{1}(\underline{y})+i \underline{v}_{2}(\underline{y})\right) f(\underline{y}) d \mathcal{H}^{2 n-1}(\underline{y}) .
$$

From (5.1) it may be concluded that $M_{1}$ and $M_{2}$ are harmonic in $\mathbb{R}^{2 n} \backslash \Sigma$ and that $M_{1} f(\infty)=M_{2} f(\infty)=0$.

By Theorem 3.2 and using (5.1), it follows that if $f$ is holomorphic in $\Omega^{+}$, then

$$
\begin{gathered}
M_{1} f(\underline{x})=f(\underline{x}), \quad \underline{x} \in \Omega^{+}, \\
M_{2} f(\underline{x})=0, \quad \underline{x} \in \Omega^{+} .
\end{gathered}
$$

Note that (5.2) corresponds to the classical Bochner-Martinelli formula, see [24].

We now assume that $\Sigma$ is an $\mathrm{AD}$-regular surface and that $f$ is a $\mathbb{C}$-valued function which belongs to $H_{\varphi}(\Sigma)$, where $\varphi$ is a regular majorant. We thus have

$$
\mathrm{S}_{\Sigma}^{\text {isot }} f(\underline{z})=N_{1} f(\underline{z})+N_{2} f(\underline{z}), \quad \underline{z} \in \Sigma,
$$

where $N_{1}$ and $N_{2}$ are the singular versions of $M_{1}$ and $M_{2}$ respectively, given by

$$
N_{1} f(\underline{z})=2 \lim _{\epsilon \rightarrow 0+} N_{1, \epsilon} f(\underline{z})+f(\underline{z})
$$

with

$$
\begin{aligned}
N_{1, \epsilon} f(\underline{z}) & \\
= & -\int_{\Sigma \backslash B(\underline{z}, \epsilon)}\left\langle E_{1}(\underline{y}-\underline{z})-i E_{2}(\underline{y}-\underline{z}), \underline{v}_{1}(\underline{y})+i \underline{v}_{2}(\underline{y})\right\rangle \\
& \times(f(\underline{y})-f(\underline{z})) d \mathcal{H}^{2 n-1}(\underline{y})
\end{aligned}
$$


and

$$
N_{2} f(\underline{z})=2 \lim _{\epsilon \rightarrow 0+} N_{2, \epsilon} f(\underline{z})
$$

with

$$
\begin{aligned}
N_{2, \epsilon} f(\underline{z})= & \int_{\Sigma \backslash B(\underline{z}, \epsilon)}\left(E_{1}(\underline{y}-\underline{z})+i E_{2}(\underline{y}-\underline{z})\right) \wedge\left(\underline{v}_{1}(\underline{y})+i \underline{v}_{2}(\underline{y})\right) \\
& \times(f(\underline{y})-f(\underline{z})) d \mathcal{H}^{2 n-1}(\underline{y}) .
\end{aligned}
$$

It is natural to ask whether the Bochner-Martinelli singular integral operator $N_{1} f$ is an involution. The following theorem provides an answer to this question (see also $[29,34])$.

Theorem 5.1 Assume that $\Sigma$ is an AD-regular surface and let $\varphi$ be a regular majorant. Then $N_{1}$ and $N_{2}$ are bounded operators mapping $H_{\varphi}(\Sigma)$ into itself. Moreover, the formulae

$$
\begin{aligned}
N_{1}^{2} f+\left[N_{2}^{2} f\right]_{0} & =f, \\
N_{1} N_{2} f+N_{2} N_{1} f+\left[N_{2}^{2} f\right]_{2} & =0, \\
{\left[N_{2}^{2} f\right]_{4} } & =0,
\end{aligned}
$$

hold for all $f \in H_{\varphi}(\Sigma)$.

Proof The proof easily follows using (5.4) as well as Theorem 3.4.

It is worth noting that the formulae above were obtained in [2] (see also [32]) for the case $n=2$.

As an application of Theorem 3.3 we obtain that $M_{1}$ and $M_{2}$ have continuous limit values on $\Sigma$ given by the formulae

$$
\begin{gathered}
M_{1}^{ \pm} f(\underline{z})=\lim _{\Omega^{ \pm} \ni \underline{x} \rightarrow \underline{z}} M_{1} f(\underline{x})=\frac{1}{2}\left(N_{1} f(\underline{z}) \pm f(\underline{z})\right), \quad \underline{z} \in \Sigma, \\
M_{2}^{ \pm} f(\underline{z})=\lim _{\Omega^{ \pm} \ni \underline{x} \rightarrow \underline{z}} M_{2} f(\underline{x})=\frac{1}{2} N_{2} f(\underline{z}), \quad \underline{z} \in \Sigma .
\end{gathered}
$$

Note that (5.5) is the Plemelj-Sokhotski formulae for the Bochner-Martinelli integral (see, e.g. $[2,7,13,14,21,23,26]$ ).

It is not difficult to show that a necessary and sufficient condition for $f$ to have a holomorphic extension to $\Omega^{+}$is that

$$
\begin{aligned}
& N_{1} f=f \text { on } \Sigma, \\
& M_{2} f=0 \text { in } \Omega^{+} .
\end{aligned}
$$


Indeed, let us suppose that $F$ is a holomorphic extension of $f$ to $\Omega^{+}$. Equalities (5.2) and (5.3) now yield (5.8) and

$$
F(\underline{x})=M_{1} f(\underline{x}), \quad \underline{x} \in \Omega^{+} .
$$

From (5.5) it follows that

$$
f(\underline{z})=\frac{1}{2}\left(N_{1} f(\underline{z})+f(\underline{z})\right), \quad \underline{z} \in \Sigma,
$$

and hence $N_{1} f=f$ on $\Sigma$. Conversely, if (5.7) and (5.8) hold, then from (5.7) we can deduce that $M_{1} f$ is a harmonic extension of $f$ to $\Omega^{+}$, while (5.8) implies that $M_{1} f$ is isotonic in $\Omega^{+}$and hence holomorphic in $\Omega^{+}$.

What is more, in the next theorem we will show that condition (5.7) is redundant.

Theorem 5.2 Let $\Sigma$ be an AD-regular surface and let $\varphi$ be a regular majorant. Suppose that $f$ is a $\mathbb{C}$-valued function which belongs to $H_{\varphi}(\Sigma)$. Then the following statements are equivalent:

(i) $f$ has a holomorphic extension to $\Omega^{+}$;

(ii) $M_{2} f=0$ in $\Omega^{+}$;

(iii) $N_{2} f=0$ on $\Sigma$;

(iv) $M_{2} f=0$ in $\Omega^{-}$.

Proof (i) $\Rightarrow$ (ii): This easily follows from (5.3).

(ii) $\Leftrightarrow$ (iii) $\Leftrightarrow$ (iv): If $M_{2} f(\underline{x})=0$ for all $\underline{x} \in \Omega^{+}$, then from (5.6) we obtain $N_{2} f=0$ on $\Sigma$, and hence $M_{2}^{-} f=0$ on $\Sigma$. Since $M_{2} f$ is harmonic in $\Omega^{-}$and vanishes at infinity, it follows that $M_{2} f(\underline{x})=0$ for all $\underline{x} \in \Omega^{-}$. In the same way we can show that (iv) $\Rightarrow$ (ii).

(iv) $\Rightarrow$ (i): Now assume that $M_{2} f(\underline{x})=0$ for all $\underline{x} \in \Omega^{-}$. From (5.1) we see that $M_{1} f$ is isotonic in $\Omega^{-}$and hence holomorphic in $\Omega^{-}$. Lemma 4.1 now shows that $M_{1} f=0$ in $\Omega^{-}$. This gives $N_{1} f=f$ on $\Sigma$, which follows from (5.5), and consequently $M_{1}^{+} f=f$ on $\Sigma$. It only remains to show that $M_{1} f$ is holomorphic in $\Omega^{+}$. This follows using (5.1) and the fact that (iv) $\Rightarrow$ (ii).

We have already proved that $M_{2} f=0$ in $\Omega^{-}$implies $M_{1} f=0$ in $\Omega^{-}$. Using our techniques, we will prove how the inverse assertion may be deduced for the case $n=2$. Indeed, if $M_{1} f=0$ in $\Omega^{-}$, then we have that $M_{2} f$ is isotonic in $\Omega^{-}$. Since $n=2$, it follows that $M_{2} f=g e_{1} e_{2}$ for some $\mathbb{C}$-valued function $g$. It is easy to check that a function of this form is isotonic if and only if the function $g$ is antiholomorphic, i.e. $\bar{g}$ is holomorphic. Therefore $M_{2} f=0$ in $\Omega^{-}$by Lemma 4.1. We have thus obtained an alternative proof of Theorem 1.1 for $n=2$.

An easy consequence of Theorem 5.2 is the following corollary.

Corollary 5.3 Let $\Sigma$ be an AD-regular surface and let $\varphi$ be a regular majorant. Suppose that $F$ is a $\mathbb{C}$-valued continuous function on $\overline{\Omega^{+}}$such that $f=\left.F\right|_{\Sigma} \in H_{\varphi}(\Sigma)$. A necessary and sufficient condition for $F$ to be holomorphic in $\Omega^{+}$is that $F$ is harmonic in $\Omega^{+}$and that $M_{2} f(\underline{x})=0$ for all $\underline{x} \in \Omega^{+}$. 
Proof If $F$ is holomorphic in $\Omega^{+}$, then obviously $F$ is harmonic in $\Omega^{+}$and $M_{2} f(\underline{x})=0$ in $\Omega^{+}$by (5.3). Now, if $M_{2} f(\underline{x})=0$ for all $\underline{x} \in \Omega^{+}$, then by Theorem 5.2 the function

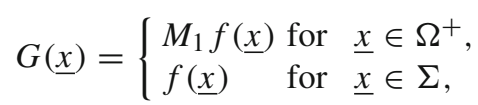

is a holomorphic extension of $f$ to $\Omega^{+}$. As $F-G$ is harmonic in $\Omega^{+}$and $\left.(F-G)\right|_{\Sigma}=0$ we have $F(\underline{x})=M_{1} f(\underline{x})$ for all $\underline{x} \in \Omega^{+}$, which follows from the maximum principle for harmonic functions.

Note that if $F=M_{1} f$ in $\Omega^{+}$, then clearly $F$ is harmonic in $\Omega^{+}$. Using (5.5) we also obtain $N_{1} f=f$ on $\Sigma$, and hence $M_{1}^{-} f=0$ on $\Sigma$. The maximum principle for harmonic functions now yields $M_{1} f=0$ in $\Omega^{-}$. This completes the proof of Theorem 1.2 for $n=2$.

It is also worth remarking that our assumptions on $f$ and $\Sigma$ are less restrictive than Aronov-Kytmanov's assumptions.

\section{Holomorphic Extension for Continuous Functions}

In Theorem 5.2 we have assumed that $f$ belongs to some space of generalized Hölder continuous functions $H_{\varphi}(\Sigma)$, with $\varphi$ a regular majorant. An obvious question to ask is whether the assertion of Theorem 5.2 continues to hold for merely continuous functions on $\Sigma$.

It is the final aim of this section to answer that question, but first we will prove that the Plemelj-Sokhotski formulae (5.5) and (5.6) are still valid for a subclass of continuous functions wider than $H_{\varphi}(\Sigma)$.

Theorem 6.1 Let $\Sigma$ be an AD-regular surface and let $f$ be a $\mathbb{C}$-valued continuous function on $\Sigma$. If the integrals

$$
\int_{\Sigma \cap B(\underline{z}, \epsilon)}\left\langle E_{1}(\underline{y}-\underline{z})-i E_{2}(\underline{y}-\underline{z}), \underline{v}_{1}(\underline{y})+i \underline{v}_{2}(\underline{y})\right\rangle(f(\underline{y})-f(\underline{z})) d \mathcal{H}^{2 n-1}(\underline{y})
$$

converge uniformly to zero for $\underline{z} \in \Sigma$ as $\epsilon \rightarrow 0$, then the Bochner-Martinelli integral $M_{1} f$ has continuous limit values on $\Sigma$ given by (5.5).

Proof We restrict ourselves to the proof of the statement for $M_{1}^{+} f$, the proof of the one for $M_{1}^{-} f$ being similar. 
Let $\underline{z}$ be a fixed point of $\Sigma$ and let $\underline{x} \in \Omega^{+}$. If $\underline{z}_{\underline{x}} \in\{\underline{y} \in \Sigma:|\underline{y}-\underline{x}|=\operatorname{dist}(\underline{x}, \Sigma)\}$, we have that

$$
\begin{aligned}
& \left|M_{1} f(\underline{x})-\frac{1}{2}\left(N_{1} f(\underline{z})+f(\underline{z})\right)\right| \leq\left|M_{1} f(\underline{x})-N_{1, \epsilon} f\left(\underline{z}_{\underline{x}}\right)-f\left(\underline{z}_{\underline{x}}\right)\right| \\
& +\mid \int_{\Sigma \cap B\left(\underline{z}_{\underline{x}}, \epsilon\right)}\left\langle E_{1}\left(\underline{y}-\underline{z}_{\underline{x}}\right)-i E_{2}\left(\underline{y}-\underline{z}_{\underline{x}}\right), \underline{v}_{1}(\underline{y})+i \underline{v}_{2}(\underline{y})\right\rangle \\
& \quad \times\left(f(\underline{y})-f\left(\underline{z}_{\underline{x}}\right)\right) d \mathcal{H}^{2 n-1}(\underline{y}) \mid \\
& +\left|N_{1} f\left(\underline{z}_{\underline{x}}\right)-N_{1} f(\underline{z})\right|+\left|f\left(\underline{z}_{\underline{x}}\right)-f(\underline{z})\right|,
\end{aligned}
$$

with $\epsilon=\operatorname{dist}(\underline{x}, \Sigma)$.

By Lemma 3.5 and using the fact that $\Sigma$ is an AD-regular surface we can deduce that

$$
\left|\mathrm{C}_{\Sigma}^{\text {isot }} f(\underline{x})-\mathrm{S}_{\Sigma, \epsilon}^{\text {isot }} f\left(\underline{z}_{\underline{x}}\right)-f\left(\underline{z}_{\underline{x}}\right)\right| \leq C\left(\omega_{f}(\epsilon)+\epsilon \int_{\epsilon}^{d} \frac{\omega_{f}(\tau)}{\tau^{2}} d \tau\right)
$$

From the above it follows that

$$
\left|M_{1} f(\underline{x})-N_{1, \epsilon} f\left(\underline{z}_{\underline{x}}\right)-f\left(\underline{z}_{\underline{x}}\right)\right| \leq C\left(\omega_{f}(\epsilon)+\epsilon \int_{\epsilon}^{d} \frac{\omega_{f}(\tau)}{\tau^{2}} d \tau\right)
$$

By the assumptions on $f$ and using the last inequality, it is easily seen that the righthand side of (6.1) tends to zero as $\underline{x} \rightarrow \underline{z}$.

We note that Theorem 6.1 was already obtained by Gaziev for sufficiently smooth surfaces in $[13,14]$.

In a similar way we can prove the following.

Theorem 6.2 Let $\Sigma$ be an AD-regular surface and let $f$ be a $\mathbb{C}$-valued continuous function on $\Sigma$. If the integrals

$$
\int_{\Sigma \cap B(\underline{z}, \epsilon)}\left(E_{1}(\underline{y}-\underline{z})+i E_{2}(\underline{y}-\underline{z})\right) \wedge\left(\underline{v}_{1}(\underline{y})+i \underline{v}_{2}(\underline{y})\right)(f(\underline{y})-f(\underline{z})) d \mathcal{H}^{2 n-1}(\underline{y})
$$

converge uniformly to zero for $\underline{z} \in \Sigma$ as $\epsilon \rightarrow 0$, then $M_{2} f$ has continuous limit values on $\Sigma$ given by (5.6).

The following results may be easily deduced from Lemma 3.5. 
Lemma 6.3 Let $f$ be a $\mathbb{C}$-valued continuous function on $\Sigma, \underline{z} \in \Sigma$ and $\epsilon>0$.

(i) If $\underline{x} \in \Omega^{+}$is such that $|\underline{x}-\underline{z}|=\epsilon / 2$, then we have that

$$
\begin{aligned}
& \left|M_{1} f(\underline{x})-N_{1, \epsilon} f(\underline{z})-f(\underline{z})\right| \\
& \leq C\left(\frac{\theta_{\underline{z}}(\epsilon)}{(\operatorname{dist}(\underline{x}, \Sigma))^{2 n-1}} \omega_{f}(\epsilon)+\epsilon \int_{\epsilon}^{d} \frac{\omega_{f}(\tau)}{\tau^{2 n}} d \theta_{\underline{z}}(\tau)\right) .
\end{aligned}
$$

(ii) If $\underline{x} \in \Omega^{-}$is such that $|\underline{x}-\underline{z}|=\epsilon / 2$, then we have that

$$
\begin{aligned}
& \left|M_{1} f(\underline{x})-N_{1, \epsilon} f(\underline{z})\right| \\
& \quad \leq C\left(\frac{\theta_{\underline{z}}(\epsilon)}{(\operatorname{dist}(\underline{x}, \Sigma))^{2 n-1}} \omega_{f}(\epsilon)+\epsilon \int_{\epsilon}^{d} \frac{\omega_{f}(\tau)}{\tau^{2 n}} d \theta_{\underline{z}}(\tau)\right) .
\end{aligned}
$$

Lemma 6.4 Let $f$ be a $\mathbb{C}$-valued continuous function on $\Sigma, \underline{z} \in \Sigma$ and $\epsilon>0$. Then we have that

$$
\left|M_{2} f(\underline{x})-N_{2, \epsilon} f(\underline{z})\right| \leq C\left(\frac{\theta_{\underline{z}}(\epsilon)}{(\operatorname{dist}(\underline{x}, \Sigma))^{2 n-1}} \omega_{f}(\epsilon)+\epsilon \int_{\epsilon}^{d} \frac{\omega_{f}(\tau)}{\tau^{2 n}} d \theta_{\underline{z}}(\tau)\right),
$$

where $\underline{x} \in \Omega^{ \pm}$is such that $|\underline{x}-\underline{z}|=\epsilon / 2$.

We remark that Lemma 6.3 have been previously proved by Gaziev for sufficiently smooth surfaces in [12].

In what follows, we will assume that $\Omega^{+}$is a Lipschitz domain, i.e. its boundary $\Sigma$ is locally the graph of a Lipschitz continuous function (see, e.g. [15]).

It is well-known that bounded Lipschitz domains satisfy the so-called uniform interior and exterior cone condition. That is, there exists constants $\lambda>0, \delta>0$ such that for every $\underline{z} \in \Sigma$, one of the two components of $V(\underline{z}) \cap\{\underline{\zeta}:|\underline{\zeta}-\underline{z}|<\delta\}$ is completely contained in $\Omega^{+}$and the other is completely contained in $\Omega^{-}$, where

$$
V(\underline{z})=\{\underline{x}:|\underline{x}-\underline{z}| \leq(1+\lambda) \operatorname{dist}(\underline{x}, \Sigma)\} .
$$

Theorem 6.5 Let $\Omega^{+}$be a Lipschitz domain. Suppose that $f$ is a $\mathbb{C}$-valued continuous function on $\Sigma$. Then $f$ has a holomorphic extension to $\Omega^{+}$if and only if $M_{2} f=0$ in $\Omega^{+}$.

Proof The necessity is obvious. Thus, we show the sufficiency. Suppose that $M_{2} f=0$ in $\Omega^{+}$. It follows that $M_{1} f$ is holomorphic in $\Omega^{+}$. Now if $\underline{z} \in \Sigma$ and $\epsilon>0$, then by Lemma 6.4 we get that

$$
\left|N_{2, \epsilon} f(\underline{z})\right| \leq C\left(\frac{\theta_{\underline{z}}(\epsilon)}{(\operatorname{dist}(\underline{x}, \Sigma))^{2 n-1}} \omega_{f}(\epsilon)+\epsilon \int_{\epsilon}^{d} \frac{\omega_{f}(\tau)}{\tau^{2 n}} d \theta_{\underline{z}}(\tau)\right)
$$


where $\underline{x} \in \Omega^{+}$is such that $|\underline{x}-\underline{z}|=\epsilon / 2$. When $\underline{x}$ approaches $\underline{z}$ non-tangentially inside the cone $V(\underline{z})$, the uniform interior and exterior cone condition implies that

$$
\frac{\epsilon}{2}=|\underline{x}-\underline{z}| \leq(1+\lambda) \operatorname{dist}(\underline{x}, \Sigma)
$$

Combining the above inequality with the $\mathrm{AD}$-regularity of $\Sigma$, we obtain

$$
\left|N_{2, \epsilon} f(\underline{z})\right| \leq C\left(\omega_{f}(\epsilon)+\epsilon \int_{\epsilon}^{d} \frac{\omega_{f}(\tau)}{\tau^{2}} d \tau\right)
$$

Therefore $N_{2, \epsilon} f(z)$ converges uniformly on $\Sigma$ as $\epsilon \rightarrow 0$. Theorem 6.2 now shows that $M_{2} f$ has continuous limit values on $\Sigma$ given by (5.6). This clearly forces $M_{2} f=0$ in $\Omega^{-}$and hence $M_{1} f=0$ in $\Omega^{-}$.

By Lemma 6.3 we thus get that

$$
\left|N_{1, \epsilon} f(\underline{z})\right| \leq C\left(\frac{\theta_{\underline{z}}(\epsilon)}{(\operatorname{dist}(\underline{x}, \Sigma))^{2 n-1}} \omega_{f}(\epsilon)+\epsilon \int_{\epsilon}^{d} \frac{\omega_{f}(\tau)}{\tau^{2 n}} d \theta_{\underline{z}}(\tau)\right),
$$

where $\underline{x} \in \Omega^{-}$is such that $|\underline{x}-\underline{z}|=\epsilon / 2$. In the same way we can see that $N_{1, \epsilon} f(\underline{z})$ converges uniformly on $\Sigma$ as $\epsilon \rightarrow 0$. Then, Theorem 6.1 implies that $M_{1} f$ has continuous limit values on $\Sigma$ given by (5.5). This gives $N_{1} f=f$ on $\Sigma$, which completes the proof.

We get the following corollary from this theorem just as we did from Theorem 5.2.

Corollary 6.6 Let $\Omega^{+}$be a Lipschitz domain. Suppose that $F$ is a $\mathbb{C}$-valued continuous function on $\overline{\Omega^{+}}$. A necessary and sufficient condition for $F$ to be holomorphic in $\Omega^{+}$is that $F$ is harmonic in $\Omega^{+}$and that $M_{2} F(\underline{x})=0$ for all $\underline{x} \in \Omega^{+}$.

Acknowledgments This paper was written when the third author was finishing his Ph.D. at the Department of Mathematical Analysis of Ghent University. D. Peña Peña was supported by a Doctoral Grant of the Special Research Fund of Ghent University.

\section{References}

1. Abreu Blaya, R., Bory Reyes, J., Peña Peña, D., Sommen, F.: The isotonic Cauchy transform. Adv. Appl. Clifford Algebr. 17(2), 145-152 (2007)

2. Abreu Blaya, R., Bory Reyes, J., Shapiro, M.: On the notion of the Bochner-Martinelli integral for domains with rectifiable boundary. Complex Anal. Oper. Theory 1(2), 143-168 (2007)

3. Aronov, A.M., Kytmanov, A.M.: The holomorphy of functions that are representable by the Martinelli-Bochner integral. Funkcional. Anal. i Priložen 9(3), 83-84 (1975)

4. Bory Reyes, J., Peña Peña, D., Sommen, F.: A Davydov theorem for the isotonic Cauchy transform. J. Anal. Appl. 5(2), 109-121 (2007)

5. Brackx, F., Delanghe, R., Sommen, F.: Clifford Analysis, Research Notes in Mathematics, vol. 76. Pitman (Advanced Publishing Program), Boston (1982) 
6. Brackx, F., De Schepper, H., Sommen, F.: The Hermitian Clifford analysis toolbox. Adv. Appl. Clifford Algebr. 18(3-4), 451-487 (2008)

7. Chen, S.J.: The boundary properties of Cauchy type integral in several complex variables. J. Math. Res. Expo. 14(3), 391-398 (1994)

8. David, G., Semmes, S.: Analysis of and on Uniformly Rectifiable Sets. Mathematical Surveys and Monographs, vol. 38. American Mathematical Society, Providence (1993)

9. Delanghe, R., Sommen, F., Souček, V.: Clifford Algebra and Spinor-Valued Functions, Mathematics and its Applications, vol. 53. Kluwer, Dordrecht (1992)

10. Falconer, K.J.: The Geometry of Fractal Sets, Cambridge Tracts in Mathematics, vol. 85. Cambridge University Press, Cambridge (1986)

11. Federer, H.: Geometric measure theory, Die Grundlehren der mathematischen Wissenschaften, Band 153. Springer, New York (1969)

12. Gaziev, A.: Limit values of a Martinelli-Bochner integral. Izv. Vyssh. Uchebn. Zaved. Mat. 9(196), 25-30 (1978)

13. Gaziev, A.: Necessary and sufficient conditions for continuity of the Martinelli-Bochner integral. Izv. Vyssh. Uchebn. Zaved. Mat. 9, 13-17 (1983)

14. Gaziev, A.: Some properties of an integral of Martinelli-Bochner type with continuous density. Izv. Akad. Nauk UzSSR Ser. Fiz.-Mat. Nauk 1, 16-22, 93 (1985)

15. Gilbert, J., Murray, M.: Clifford Algebras and Dirac Operators in Harmonic Analysis, Cambridge Studies in Advanced Mathematics, vol. 26. Cambridge University Press, Cambridge (1991)

16. Gunning, R.C.: Introduction to Holomorphic Functions of Several Variables, 3 vols. Wadsworth \& Brooks/Cole Advanced Books \& Software, Monterey (1990)

17. Gürlebeck, K., Sprössig, W.: Quaternionic and Clifford Calculus for Physicists and Engineers. Wiley, New York (1997)

18. Guseřnov, A.I., Muhtarov, H.Š.: Introduction to the theory of nonlinear singular integral equations, "Nauka", Moscow (1980)

19. Hörmander, L.: An Introduction to Complex Analysis in Several Variables, 3rd edn. North-Holland Mathematical Library, vol. 7. North-Holland, Amsterdam (1990)

20. Krantz, S.G.: Function Theory of Several Complex Variables, 2nd edn. The Wadsworth \& Brooks/Cole Mathematics Series, Wadsworth \& Brooks/Cole Advanced Books \& Software, Pacific Grove (1992)

21. Kytmanov, A.M.: The Bochner-Martinelli Integral and its Applications. Birkhäuser, Basel (1995)

22. Kytmanov, A.M., Aĭzenberg, L.A.: The holomorphy of continuous functions that are representable by the Martinelli-Bochner integral. Izv. Akad. Nauk Armjan. SSR Ser. Mat. 13(2), 158-169, 173 (1978)

23. Ma, Z.T., Zhang, Q.Q.: Boundary properties of Bochner-Martinelli type integrals. Pure Appl. Math. (Xi'an) 18(4), 313-316 (2002)

24. Martinelli, E.: Sulle estensioni della formula integrale di Cauchy alle funzioni analitiche di più variabili complesse. Ann. Mat. Pura Appl. 34(4), 277-347 (1953)

25. Mattila, P.: Geometry of Sets and Measures in Euclidean Spaces, Fractals and Rectifiability, Cambridge Studies in Advanced Mathematics, vol. 44. Cambridge University Press, Cambridge (1995)

26. Mitelman, I.M., Shapiro, M.V.: Differentiation of the Martinelli-Bochner integrals and the notion of hyperderivability. Math. Nachr. 172, 211-238 (1995)

27. Range, R.M.: Holomorphic Functions and Integral Representations in Several Complex Variables, Graduate Texts in Mathematics, vol. 108. Springer, New York (1986)

28. Range, R.M.: Complex analysis: a brief tour into higher dimensions. Am. Math. Mon. 110(2), 89-108 (2003)

29. Rocha Chávez, R., Shapiro, M., Sommen, F.: On the singular Bochner-Martinelli integral. Integral Equ. Oper. Theory 32(3), 354-365 (1998)

30. Rocha Chávez, R., Shapiro, M., Sommen, F.: Integral Theorems for Functions and Differential Forms in $\mathbb{C}^{m}$, Research Notes in Mathematics, vol. 428. Chapman \& Hall/CRC, Boca Raton (2002)

31. Sabadini, I., Sommen, F.: Hermitian Clifford analysis and resolutions. Math. Methods Appl. Sci. 25 (16-18), 1395-1413 (2002)

32. Schneider, B.: On the Bochner-Martinelli operator. Appl. Comput. Math. 4(2), 200-209 (2005)

33. Sommen, F., Peña Peña, D.: Martinelli-Bochner formula using Clifford analysis. Archiv. Math. 88(4), 358-363 (2007)

34. Tarkhanov, D.: Operator algebras related to the Bochner-Martinelli integral. Complex Var. Elliptic Equ. 51(3), 197-208 (2006) 